\title{
Maternal gestures with 20-month-old infants in two contexts
}

\section{Maria O’Neill, Kim A. Bard, Maggie Linnell and Michael Fluck}

Department of Psychology, University of Portsmouth, UK

Address for correspondence: Maria O'Neill, Centre for Psychology Studies, University of

Bradford, Bradford BD7 1DP, UK; e-mail: maria.1.oneill@gmail.com

\begin{abstract}
Speech directed towards young children ('motherese') is subject to consistent systematic modifications. Recent research suggests that gesture directed towards young children is similarly modified (gesturese). It has been suggested that gesturese supports speech, therefore scaffolding communicative development (the facilitative interactional theory). Alternatively, maternal gestural modification may be a consequence of the semantic simplicity of interaction with infants (the interactional artefact theory). The gesture patterns of 12 English mothers were observed with their 20-month-old infants while engaged in two tasks, free play and a counting task, designed to differentially tap into scaffolding. Gestures accounted for $29 \%$ of total maternal communicative behaviour. English mothers employed mainly concrete deictic gestures (e.g. pointing) that supported speech by disambiguating and emphasizing the verbal utterance. Maternal gesture rate and informational gesture-speech relationship were consistent across tasks, supporting the interactional artefact theory. This distinctive pattern of gesture use for the English mothers was similar to that reported for American and Italian mothers, providing support for universality. Child-directed gestures are not redundant in relation to child-directed speech but rather both are used by mothers to support their communicative acts with infants.
\end{abstract}

A great deal of recent research has focused on the differences between adult-adult and adultchild interactions. Child-directed speech, gesture and motion are all subject to systematic modification, relative to adult-directed speech, gesture and motion. The precise nature of the modifications in childdirected speech ('motherese') is well documented (see Snow, 1995, for a review). Mothers also modify child-directed actions on objects ('motionese'), compared with actions on the same object during inter- 
action with adults (Brand, Baldwin \& Ashburn, 2002). Comparable consistent, systematic modifications are found in child-directed gesture ('gesturese') (Bekken, 1989; Iverson, Capirci, Longobardi \& Caselli 1999; O’Neill, 2003). The aim of the current study is to identify and describe the communicative gestures of English mothers during interaction with 20-month-olds during two tasks, a counting task and a free play task.

Previous research has highlighted two probable features of gesturese which are modified relative to adult-directed gesture: (1) mothers tend to used fewer gestures with children than with adults (e.g. Bekken, 1989); (2) mothers tend to employ deictic gestures rather than emphatic or iconic gestures during interaction with children (e.g. Iverson et al., 1999; Shatz, 1982). Typically, gesturese can be characterized by fewer of the fluid hand waving emphatic gestures typical of adult-adult interactions, a larger number of conceptually simple gestures (i.e. pointing) (Bekken, 1989; Iverson et al., 1999; Schmidt, 1996; Shatz, 1982); on the other hand, gesturese appears tied to context, typically comprises concrete gesture and can be characterized as a limited gestural repertoire. Finally, gesturese is typically employed alongside utterances (e.g., 'look at the doggy' + point to the dog).

There is some evidence that gesturese may be univer- sal. Iverson et al. (1999) described gesturese produced by Italian mothers, and categorized the gesture-speech rela- tionship. Like American mothers (Shatz, 1982; Bekken, 1989; Schmidt, 1996), Italian mothers typically employ many deictic gestures, serving to reinforce accompanying speech. Italian mothers accompany approximately $15 \%$ of verbal utterances to 16-20-month-old infants with gesture, while American mothers use gesture alongside approximately $43 \%$ of speech to 19 -month-old children, and $23 \%$ of speech to 34-month-olds.

Evidence suggests that maternal proportional gesture rates vary according to the child's age (Shatz, 1982), linguistic ability (Iverson et al., 1999), and comprehension of a message (Schmidt, 1996). To control for a potential confound between age and task, the current study focuses on 
children matched for age. Twenty-month-olds were among the youngest in the Shatz study, eliciting the highest proportion of maternal gesture, and were the oldest in Iverson et al.'s study, also eliciting the highest proportional gesture rates. Therefore 20-month-olds were expected to elicit a high rate of maternal gesture, and provide the most appropriate aged subjects for cross-cultural comparison.

The current study extends the previous research in several ways. Previous research was conducted in America (Bekken, 1989; Schmidt, 1996; Shatz, 1982) and Italy (Iverson et al., 1999). Striking similarities were found in the gestures employed by mothers interacting with their young children, despite the very different cultural norms associated with adult-adult gestures. However, previous research has tended to focus on mother- child interaction during free play only, and little is known about the variability of maternal gesture across contexts. The primary aim of the current study is to identify and describe the gesture style of English mothers during interaction with 20-month-olds during two tasks that differentially tap scaffolding, a counting task and a free play task. Specifically, we were interested in the effect that engaging in a structured counting task might have on maternal gesture. Structured counting tasks elicit greater scaffolding efforts from mothers (Fluck, 1995; 1993; Fluck \& Maltby, 1997; Linnell \& Fluck, 2000) and elicit gestures from children which influence their performance (Alibali \& DiRusso, 1999; Saxe \& Kaplan, 1981). Our research question concerns the way gesture is used in a structured counting task compared with free play. The structured counting task has a clearly defined goal, as one expert (the mother) helps a novice (the child) to count several objects. The task is expected to elicit scaffolding from the mothers, as they help the child work towards a clearly defined goal. The free play task has no clear goal, and is therefore symmetrical with respect to goal, but not necessarily with respect to linguistic ability. Because the tasks were designed to differ in the necessity for maternal scaffolding, we expected to be able to differentiate between two theories regarding the function of maternal gesture, the "facilitative interaction' hypothesis, and the 'interactional artefact' hypothesis. 
The communicative environment experienced by children is thought to support emergent infant ability: motherese facilitates lexical and syntactic development and general communicative skill (see Snow, 1995): motionese facilitates infant attention, enhancing learn- ing and /or comprehension of action (Brand et al., 2002); similarly, Iverson et al. (1999) argue that gesturese serves to initiate and maintain the child's attentional focus. The co-use of gesturese alongside and reinforcing motherese may further attract and maintain attention, facilitating joint attentional focus. Therefore we predict that maternal gesture will be redundant with co-occurring speech.

There are two principal explanations for the existence of maternal modification. Under the 'Interactional Artefact' hypothesis, largely associated with Pine (1994), modification of child-directed communication is a by- product of communication restricted to talk of the 'here and now', occurring from the semantic simplicity of interaction with children. Given the context dependent nature of childdirected communicative acts, we might expect that gesture would serve to reinforce - rather than to disambiguate or add to - speech. It is plausible to argue that a message comprising speech with gesture providing the same message as speech is simpler to comprehend than speech with gesture providing conflicting or different information. Conversely, under the 'Facilitative Strategy' hypothesis, modification of child-directed communication exists to assist language learning in children, a supportive strategy serving to facilitate language learning. Gesture serving to disambiguate speech would seem likely in the context of the facilitative strategy hypothesis because gestures that assist the child by indicating the precise referent of speech (i.e. disambiguate the referent) would support the acquisition of lexical items.

The two different views of maternal modification, as a facilitative strategy versus an interactional artefact, have important implications for the current research. If maternal modifications serve to facilitate or scaffold emergent ability, then mothers would be expected to adjust communicative behaviour when the child requires additional support. However, if maternal 
modification is merely an interactional artefact, then contextual changes should have little effect on maternal communicative style.

The current study was designed to describe the nature of maternal gesture during interaction with 20-month-old infants, explore differences in maternal gesture patterns between two tasks, and compare the pattern observed in this English sample with that found in America and Italy. Any difference between gesture patterns in the two contexts would support the notion that maternal modification has a facilitative role in scaffolding emergent ability. Alternatively, the absence of a difference would support the notion that modification is an interactional artefact resulting from the semantic simplicity and concrete nature of child-directed speech and gesture.

\section{Method}

\section{Participants}

Twelve mothers living in southern England were selected from a longitudinal study that investigated the effect of maternal support on the development of counting and cardinal understanding of number (Linnell, 1998; Linnell \& Fluck, 2001). All mothers were full-time caregivers. The children (five girls) were 20 months old ( \pm 1 week) and were developing typically.

\section{Procedure}

Mother-child dyads were videotaped for two 5-minute sessions, a free play session and a counting task. Observations took place in a comfortably furnished university- based observation laboratory. The dyads were alone in the room throughout each session.

Free play session

The dyads were videotaped while engaging in a range of self-selected activities, such as 
drawing, puzzles and duplo@ bricks. The majority of dyads (nine dyads) selected picture books to read. Dyads were provided with refreshments during the free play session. Mothers were given no explicit instructions regarding their interactions.

Structured count task

In this session, the child was asked to count the toys in each of three baskets. The baskets, containing two, three or seven toys, were individually presented to the child, in a random order, with the constraint that the basket with seven toys was not presented first. Mothers were instructed to give as much or as little help and encourage- ment as they felt their child needed (see Linnell \& Fluck, 2001, for the rationale for the count task).

\section{Coding and analysis}

The videotaped observations were coded with a scheme adapted from Iverson et al. (1999). Each maternal communicative act was classified in one of three mutually exclusive and exhaustive categories: gesture alone, speech alone, or gesture and speech. A speech utterance was defined as any verbalization followed by a silence, change in conversational turns, or change in intonation pattern, following criteria used by Iverson et al. (1999). A gesture utterance was defined as a hand, head, arm or body movement, preceded and followed by a clear pause or relaxation of (e.g.) hand, arm or head

position, following criteria used by Namy, Acredolo and Good- win (2000). Gesture-speech cooccurrence was defined as any occasion when a gesture was enacted during a speech utterance. The communicative meaning of each gesture was further coded in one of four categories: deic- tic (with four subtypes), conventional, representational and emphatic (see below). Additionally, when gesture and speech co-occurred, the informational relation was classified into one of four categories: emphasizing, disambiguating, adding and contradicting (see below). 
Types of maternal gesture

All occurrences of maternal gesture were coded accord- ing to the communicative meaning of the gesture. Deictic gestures indicated the existence of an object, person or occurrence of an event. The following four subtypes of deictic gestures were coded. A deictic gesture was coded as 'point' when a finger was extended towards an object or event. The gestures coded as 'show' were instances when an object was held up in the gesture space and oriented towards the child. Gestures deemed to 'indicate' were those where an object or event was singled out through movements of the head or hand towards the object or event, or direct contact with (i.e. tapping or touching) the object or location of the event. Finally, the category of 'give' included those gestures where an object was handed to the child, or placed into his or her gestural space (e.g. placing an object on the table directly in front of the child).

Gestures whose form and meaning are culturally defined and therefore consistent across time were coded as conventional gestures; examples include shaking the head from side to side to indicate 'no', and beckoning with either the whole hand or the index finger. Gestures which referred to objects, locations, individuals or events were deemed representational gestures. Such gestures are used to describe an attribute or action of an object, and differ from deictic gestures, as their meaning does not change across situations. An example of this is to perform the action of brushing the teeth.

The fourth category of communicative meaning in maternal gestures was emphatic. Emphatic gestures high- light aspects of discourse structure and/or the content of accompanying speech. They are non-representational, have no specific semantic content or precise referent, and are not linked to a specific hand shape or facial expression. Nodding the head while stressing a particular word or sentence would be an emphatic gesture, as would the making of circular motions with a flat hand during speech. These emphatic, or beat, gestures are typically executed in a rhythmic fashion during adult-adult speech (McNeill, 1992), but are not very commonly reported in 
adult- child speech (Iverson \& Fagan, 2004).

Informational relationship of gesture to speech

Gestures that co-occurred with speech were placed into one of four categories according to the informational relation between gesture and speech. A gesture was emphasizing the verbal utterance if the gesture was semantically equivalent and conveyed the same message as speech (in other words, an emphasizing gesture reinforces speech): e.g. saying 'no' while shaking the head from side to side. A gesture that identified the precise referent of a verbal utterance, serving to clarify and disambiguate the verbal portion of utterance, was disambiguating. Uttering a number in a count sequence and simultaneously gesturing towards the corresponding object was classified as a disambiguating gesture. Those gestures that provided information not conveyed in the accompanying utterance were seen to be adding information. Examples include saying 'sss' while pointing to a snake. Gestures conveying information discordant with accompanying speech were contradicting. Contradicting gestures differ from adding gestures as the gestural information is unrelated to the verbal utterance, e.g. uttering a number in a count sequence and simultaneously beckoning the child.

\section{Reliability}

Inter-observer reliability measures were obtained on $10 \%$ of the videotaped observations. Inter-observer agreement for identifying instances of maternal gesture and for identifying gesture by type was $97 \%(N=219)$. Agreement for identifying the informational relationship between gesture and speech was $98 \%(N=212)$.

\section{Results}


Maternal communicative acts

Maternal gesture rates were relatively high during inter- action with 20-month-olds (free play mean frequency $=6.5$ times per minute, $\mathrm{SD}=4.2$; counting mean frequency $=7.2, \mathrm{SD}=2.4)($ see Figure 1). Gestural communication accounted for $29 \%$ of total maternal communication during the free play session, and $28 \%$ of total maternal communication during the count task. Most communicative acts, however, consisted of speech alone (free play, $M=116.58, \mathrm{SD}=37.68$; counting, $M=135.83, \mathrm{SD}=60.61$ ). Neither the frequency of gesture alone (free play, $M=0.67, \mathrm{SD}=0.89$; counting, $M=1.17, \mathrm{SD}=1.6$ ) or the frequency of gesture with speech (free play $M=$ 33.75, $\mathrm{SD}=20.72$; counting, $M=42.66, \mathrm{SD}=18.76)$ differed between the play and count sessions. There were no significant differences in rate or frequency of maternal gesture or speech production across tasks: no significant differences were found between play and count session in: rate of gesture with speech $t(11)=.50, p=.63$, ns; frequency of gesture with speech $t(11)=.55, p=.59$, ns; rate of speech alone $t(11)=-1.56, p=.15$, ns; frequency of speech alone $t(11)=-1.36, p=.20$, ns.

Table 1 Individual differences in use of gesture to support speech

Occurrences of gestures serving to support speech in each way: Total for each dyad across tasks

\begin{tabular}{llllllllllllllllllllllll}
\hline Dyad & A & B & C & D & E & F & G & H & I & J & K & L
\end{tabular}

$\begin{array}{lllllllllllll}\text { Disambiguating } & 111 & 7 & 53 & 71 & 101 & 37 & 37 & 44 & 108 & 48 & 51 & 22\end{array}$

$\begin{array}{lrrrrrrrrrrrrr}\text { Emphasizing } & 18 & 30 & 10 & 8 & 19 & 5 & 7 & 14 & 6 & 2 & 5 & 1\end{array}$

$\begin{array}{lllllllllllll}\text { Adding } & 0 & 33 & 7 & 5 & 4 & 1 & 2 & 1 & 0 & 0 & 4 & 0\end{array}$

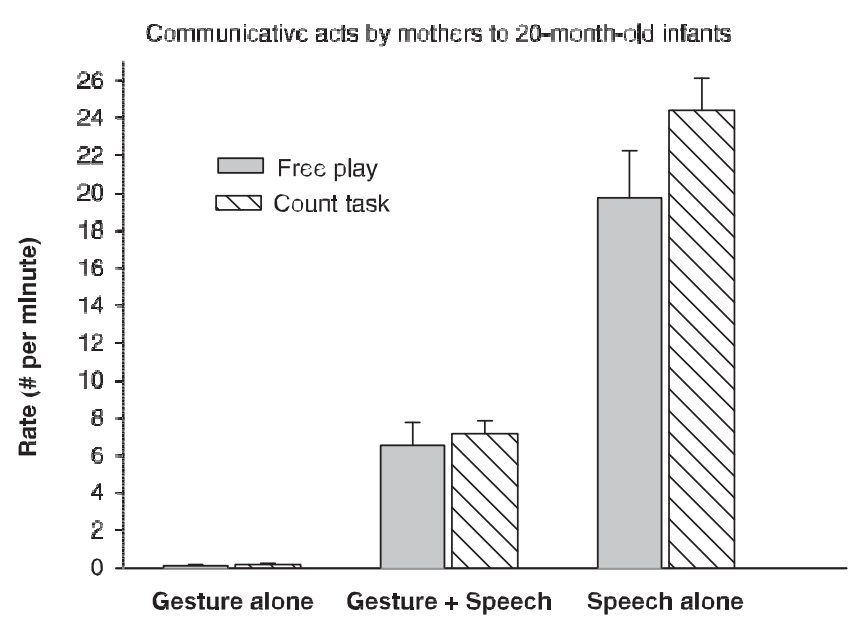

Figure 1 Maternal communicative acts across tasks. 


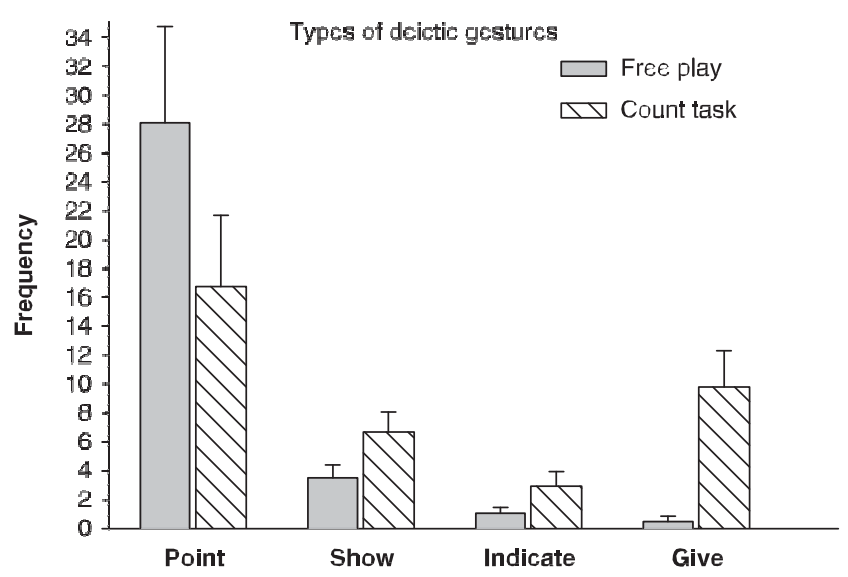

Figure 2 Types of deictic gestures.

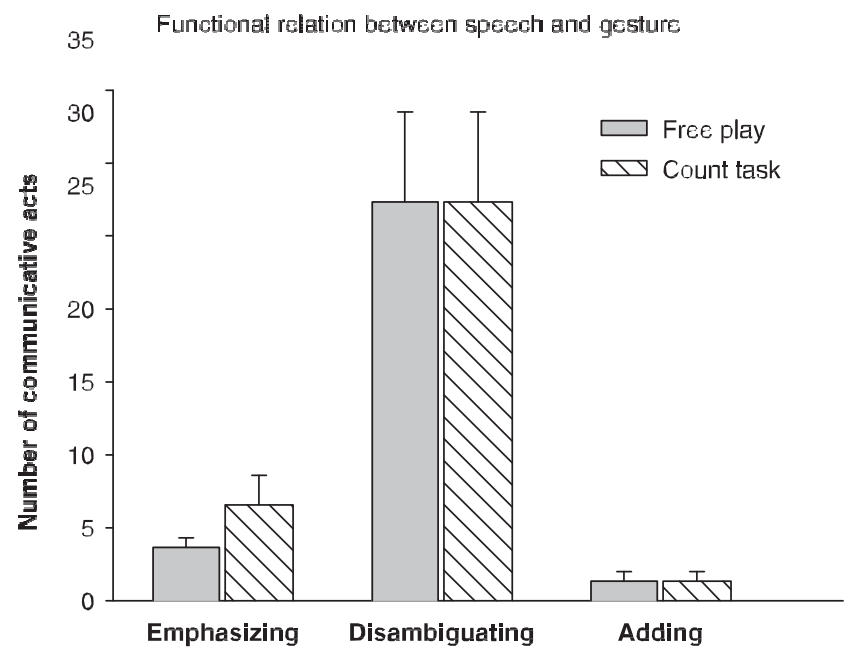

Figure 3 Gesture-speech relationship.

Types of maternal gesture

Free play session

The majority (97\%) of gestures produced by mothers in the free play session were deictic gestures $($ mean frequency $=33.3, \mathrm{SD}=20.9)$, followed by emphatic gestures $($ mean frequency $=8.33$, $\mathrm{SD}=0.29)$. Conventional gestures (mean frequency $=0.67, \mathrm{SD}=0.98$ ) and representational gestures (mean frequency $=0.25, \mathrm{SD}=0.45$ ) were used infrequently. Four different types of deictic gestures were coded (see Figure 2). Mothers tended to use many point gestures $(M=28.17, \mathrm{SD}=$ $22.97)$, and considerably fewer show $(M=3.5, \mathrm{SD}=3.06)$, give $(M=1.16, \mathrm{SD}=2.36)$ and indicate $(M=1.08, \mathrm{SD}=1.3)$ gestures during the free play session.

Count task

During the count task, deictic gestures $(M=36.83, \mathrm{SD}=22.94)$ were the most frequent type (91\%), followed by emphatic gestures $(M=2.58, \mathrm{SD}=5.79)$ and conventional gestures $(M=0.92$, $\mathrm{SD}=1.16$ ). Mothers did not use representational gestures at all during the count task. Among the 
four types of deictic gestures (Figure 2), mothers tended to use many point gestures $(M=17.0, \mathrm{SD}$ $=17.5)$, fewer show $(M=8.92, \mathrm{SD}=6.92)$, give $(M=7.92, \mathrm{SD}=7.4)$ and indicate $(M=3.0, \mathrm{SD}$ $=3.43$ ) gestures.

Comparison across contexts

Analysis of the types of deictic gestures, using the Wilcoxon signed ranks test revealed no significant effect of task on the frequency of point gestures. However, a significant effect of task was found on the frequency of indicate gestures, $z(12)=1.97, p=0.049$, show gestures, $z(12)=$ 2.32, $p=0.020$, and give gestures, $z(12)=2.18, p=0.029$. Mothers used significantly more indicate, show and give gestures during the count task than during the free play session (Figure 2).

\section{Maternal gesture and speech relationship}

The majority of gestures used by mothers were deictic, pointing gestures. These gestures tended to disambiguate the verbal utterance by highlighting the precise referent (free play, $M=25.75, \mathrm{SD}$ $=22.14$; count task, $M=31.75, \mathrm{SD}=18.85)$. The remaining portion of gestures served to add information to (free play, $M=4.08, \mathrm{SD}=9.40$; count task, $M=6.58, \mathrm{SD}=6.98$ ), or emphasize the verbal utterance (free play, $M=3.75, \mathrm{SD}=2.22$; count task, $M=0.75, \mathrm{SD}=1.48$ ). There were no significant differences found across tasks (Figure 3). Only one occurrence of a gesturespeech contradiction was observed, during the count task.

\section{Individual differences}

Overall, gesture rate varied considerably between mothers, with total proportional gesture rates across tasks varying from $14 \%$ to $37 \%$ (within free play between $12 \%$ and $41 \%$, and within the counting task between $10 \%$ and $41 \%$ ). There was no consistent pattern of differences in 
proportional gesture rates across tasks, as half of mothers exhibited a higher proportional gesture rate during the free play session, and half during the count task.

Despite the differences in gesture rate, a consistent pattern of gesture types was evident. In all cases mothers used deictic gestures most often. Three of the 12 mothers used only deictic gestures and no other gesture type, five tended to use emphatic gestures as the next most frequent category, while three used conventional gestures, and one used representational gestures as the next most frequent category.

The informational gesture-speech relationship was more consistent among mothers than the type of gesture used. The similarity in the way each mother used gesture to support speech can clearly be seen in Table 1 . Of the 12 mothers, 11 showed the identical pattern of gesture- speech relationships: gestures mainly disambiguated speech, some gestures emphasized speech but relatively fewer were used to add to speech. Only one mother (B) exhibited a different pattern, often using gesture to add to speech, used fewer gestures to emphasize speech, and relatively few of her gestures served to disambiguate speech.

\section{Discussion}

The child-directed gesture employed by mothers in the current study was similar to that identified by previous researchers, supporting the existence of a gesture style specific to interaction with children. English mothers, like American (Bekken, 1989; Schmidt, 1996; Shatz, 1982) and Italian mothers (Iverson et al., 1999), use a recognizable gesture style during interaction with young children. Gesture co-occurring with speech tended to clarify and disambiguate the referent, rather than emphasize or add meaning to the verbal utterance. Most gestures consisted of points, regardless of context. Comparison of gesture rate, and the informational gesture-speech relationship between the two tasks, counting and free play, revealed few differences, thereby failing to support 
the thesis that maternal gesture varies in relation to level of support required by children.

\section{Is child-directed gesture subject to specific modifications?}

Child-directed gestures differ systematically from adult- directed gestures. Mothers use fewer of the fluid hand waving (or beat) gestures typical of adult-adult communication; indeed, very few of the child-directed gestures in the current study were beats. Child-directed gestures tend to be concrete, conceptually simple gestures tied to the context. In the current study, points accounted for over $90 \%$ of maternal gesture. Mothers participating in the current research showed a highly consistent style of gesture within and across tasks. The significant differences in the amount of deictic gesture types indicate, show and give across tasks, are best explained as evidence of a task affordance effect: the count task affords the use of give, indicate and show gestures, while the free play task does not. Across tasks, mothers typically employed concrete, deictic gestures, particularly point gestures, which are tied to the context and conceptually simple. This adds further weight to the argument for a specific form of modification to child-directed gesture, since it demonstrates that although maternal gesture rate is variable, the pattern is constant.

These English mothers accompanied over $25 \%$ of their speech with gesture, which is higher than the $15 \%$ reported for Italian mothers (Iverson et al., 1999). This seems surprising, given that Italian is a gesture-rich culture. Even higher maternal gesture rates $(43 \%$ when the children were aged 19 months) were found in American mothers (Shatz, 1982). Both the current research and the Shatz study were conducted in English-speaking countries, suggesting some language-specific difference. American mothers tend to use a highly didactic style of child-directed speech (de Boysson-Bardies, 2001). English- speaking mothers may simply be more didactic than Italian-speaking mothers. The high gesture rate of English and American mothers consisted almost entirely of deictic gestures, indicative of a didactic communicative style. A more extensive 
cross-cultural study, examining the stability of maternal gesture rate across a wider range of linguistic cultures, would allow us to tease apart the effect of maternal speech style on gesture rate. Despite the difference in reported proportional gesture rates, however, striking similarities were found in the pattern of gestures used by mothers in all studies. This suggests that the type of gestures used by mothers, and the way those gestures augment speech, may be more important (and is certainly more consistent) than the amount of gesture used.

The informational relationship between maternal speech and gesture during the current research was also highly consistent. Mothers used gesture most often to disambiguate the verbal utterance, less often to emphasize speech and relatively infrequently to add to speech. The findings of the current research differ from those of previous researchers regarding the informational gesture-speech relationship. Iverson et al. (1999) found that Italian mothers used gesture most often to reinforce speech, while mothers in the current study used gesture most often to disambiguate speech. As mentioned previously, it is possible that this difference is due to a genuine difference in the way Italian mothers and English-speaking mothers use gesture alongside speech. Alternatively, differences between the reported gesture styles of Italian and English mothers may result from the methodological differences of each study. Mother- child dyads engaged in free play throughout Iverson et al.'s study, while the present participants engaged in both a count task and a free play session. Because most dyads in the current study elected to read books, both tasks were similar in terms of the level of instruction mothers provided. Mothers imposed a structure and goal (to read books) on the free play session, which may have elicited more scaffolding than normally seen in unstructured interactions. Mothers may use gesture to disambiguate rather than reinforce speech during instructional interactions with clearly defined goals, while the reverse may be true in less instructional contexts with no clear goal in mind. Further research investigating maternal gesture and speech across a range of contexts, which may address this issue, is in progress (O'Neill, Schafer \& 
Murray, in preparation).

Did maternal gesture differ as a function of 'task'?

We described two alternative explanations for the exist- ence of gesturese, the 'interactional artefact' hypothesis and the 'facilitative strategy' hypothesis. In the context of the interactional artefact hypothesis, we predicted that gesturese would not vary across contexts, because gesturese is symptomatic of the simplistic nature of child-directed interaction. Alternatively, under the facilitative strategy hypothesis, we predicted that the two contexts would elicit different gesture styles, because child-directed gestures and their informational relation to speech serve to facilitate linguistic development. No significant differences across tasks were found in the total of maternal utterances, total maternal gestures or the proportion of maternal speech accompanied by gesture. Gestures were used independently of the structure of the task (i.e. independent of whether it is deemed to be an 'educational' task or free play). This suggests that the structure of activity in which dyads engage does not significantly influence maternal utterance or gesture rates, supporting the interactional artefact hypothesis. To clarify this, a brief description of typical maternal behaviour during each task follows.

\section{Count task}

Mothers employed a consistent strategy to 'teach' the children to count. Mothers paired each number in a count sequence with a gesture indicating the object to be counted, thereby demonstrating one-to-one object-number relations and scaffolding the children's numerical understanding. Mothers used gesture to disambiguate speech by pairing a word (in this case a number) with gesture indicating a specific object. Mothers may use gesture alongside speech in this way to support the acquisition of not only numerosity, but also vocabulary in general (e.g. Baldwin, 1995; 
Colas, 1999; Namy et al., 2000).

Free play session

Mothers used similar strategies during the free play ses- sion, pairing a word with a gesture (such as a point), with gestures disambiguating speech by indicating the precise referent. Although the free play session was intended as an unstructured task, mothers often imposed struc- ture on these sessions by choosing to share books. While many of the words and gestures used by mothers were nouns paired with points, mothers also tended to count objects in the books while pointing to them, scaffolding both object-word pairings and numerosity.

Mothers treated both contexts as instructional situ- ations; therefore it would seem that mothers exhibit a consistent gesture style, and use gesture to support speech in specific ways, during instructional interactions. The context-dependent nature of maternal speech and gesture, paired with explicit attempts to teach vocabulary and numerosity, seem to predict that gesture be deictic and disambiguating. In essence, this typifies child-directed communication, speech disambiguated by deictic gesture. In this way, speech and gesture are used to support 'teaching' of object-word relationships to another less familiar with them.

Speech and gesture which correspond informationally (are matched) aid comprehension to a greater degree than speech and gestures which are mismatched (Goldin-Meadow, 2000; Kelly, 2001). Only one instance of a gesture-speech contradiction was observed. This suggests that children easily comprehended maternal gesture production. How- ever, as no measure of the children's comprehension was taken, it is not possible to support this assertion. Gesturese tended to be simple, brief, tied to the context and to an extent redundant. We focused on children at one age point; therefore it is not possible to speculate on how gesturese may change as the child develops linguistically. Previous research suggests that gesturese is contingent on the linguistic level of the 
interlocutor (Shatz, 1982; Iverson et al., 1999). Research is currently in progress that examines the child-directed speech and gesture of English mothers with 16-24-month-olds, the speech and gesture production of the children, and the adult-directed speech and gestures of those mothers (O'Neill, Schafer \& Murray, in preparation). In this way, it is possible that the shortcomings of this research may be overcome, and further investigation of this fascinating and important area of child development be undertaken.

Future research might consider whether gesturese is employed by all individuals, or is specific to mother-child interaction. Evidence that motherese is employed by all adults interacting with young children, by older children interacting with younger children (Shatz \& Gelman, 1973) and in communication with less compe- tent language users suggests that gesturese is not specific to mother-child interaction.

The current research describes a style of gesture employed by English mothers during interaction with 20-month-olds that is consistent across contexts. The gesturese of English mothers is comparable to gesturese observed in Italian and American mothers, supporting claims of universality of gesturese. The gesturese observed, and its relation to speech in three cultures (English, American and Italian), was comparable to motherese in terms of its brevity, simplicity and redundancy. Along with the recent finding that child-directed action is also modified (Brand et al., 2002), it seems that adults consistently modify all behaviour during interaction with infants. These modifications may exist to facilitate adult-infant interaction, or to support infant development, or both. The cross-cultural evidence strongly supports the existence of a pattern of consistent gestural modifications specific to communication with young children, termed here 'gesturese'. The 'gesturese', which consists of mainly concrete deictic gestures such as points serving to disambiguate speech by indicating the precise referent, was found to exist in English, Italian and American mothers. Furthermore, comparison of maternal gesture during a free play session and 
a count task showed that the pattern of modifications was consistent across tasks.

\section{References}

Alibali, M.W., \& DiRusso, A.A. (1999). The function of gesture in learning to count: more than keeping track. Cognitive Development, 14 (1), 37-56.

Baldwin, D.A. (1995). Understanding the link between joint attention and language. In C. Moore \& P.J. Dunham (Eds.), Joint attention: Its origins and role in development. Hove: Lawrence Erlbaum Associates.

Bekken, K. (1989). Is there motherese in gesture? Unpublished doctoral dissertation, University of Chicago, Chicago.

Brand, R.J., Baldwin, D.A., \& Ashburn, L.A. (2002). Evidence for 'motionese': modifications in mothers' infant-directed action. Developmental Science, 5 (1), $72-83$.

Colas, A. (1999). Introducing infants to referential events: a developmental study of maternal ostensive marking in French. Journal of Child Language, 26, 113-131.

de Boysson-Bardies, B. (2001). How language comes to children. London: MIT Press.

Fluck, M. (1993). The dyadic construction of number. Paper presented at the BPS Developmental Section Annual Conference, University of Birmingham.

Fluck, M. (1995). Counting on the right number. Irish Journal of Psychology, 16, 133-149.

Fluck, M., \& Maltby, M. (1997). Social factors in early number development. Paper presented at the 27th Symposium of Jean Piaget Society, Santa Monica, CA.

Goldwin-Meadow, S. (2000). Beyond words: the importance of gesture to researchers and learners. Child Development, 71 (1), 231-239.

Iverson, J.M., Capirci, O., Longobardi, E., \& Caselli, M.C. (1999). Gesturing in mother- child interactions. Cognitive Development, 14 (1), 57-75. 
Iverson, J., \& Fagan, M. (2004). Infant vocal-motor coordination: precursor to the gesture-speech system?

Child Development,

75 (4), $1053-1066$.

Kelly, S.D. (2001). Broadening the units of analysis in communication: speech and nonverbal behaviours in pragmatic comprehension. Journal of Child Language, 28, 325-349.

Linnell, M., \& Fluck, M. (2000). The effect of maternal sup- port for counting and cardinal understanding in preschool children. Social Development, 10 (2), 202-220.

McNeill, D. (1992). Hand and mind: What gestures reveal about thought. London: University of Chicago Press.

Namy, L.L., Acredolo, L., \& Goodwyn, S. (2000). Verbal labels and gestural routines in parental communication with young children. Journal of Nonverbal Behavior, 24 (2), 63-79.

O’Neill, M. (2003, 24-27 April). Maternal modification in gesture as a function of partner and context. Paper presented at the Society for Research in Child Development Biennial Meeting, Tampa, FL.

O’Neill, M., Schafer, G., \& Murray, L. (in preparation). A case for 'gesturese': consistent, systematic modifications in maternal gesture to 16-24-month-olds.

Pine, J. (1994). The language of primary caregivers. In C.R. Gallaway (Ed.), Input and interaction in language acquisition (pp. 15-37). Cambridge: Cambridge University Press.

Saxe, G.B., \& Kaplan, R. (1981). Gesture in early counting: a developmental analysis. Perceptual and Motor Skills, 53, 851-854.

Schmidt, C.L. (1996). Scrutinizing reference: how gesture and speech are co-ordinated in mother- child interaction. Journal of Child Language, 23, 297-305.

Shatz, M. (1982). On mechanisms of language acquisition: can features of the communicative environment account for development? In E.G. Wanner (Ed.), Language acquisition: The state of the art (pp. 102127). New York: Cambridge University Press. 
Shatz, M., \& Gelman, R. (1973). The development of communication skill: modifications in the speech of young children as a function of listener. Monographs of the Society for Research in Child Development, 38 (5).

Snow, C. (1995). Issues in the study of input: finetuning, universality, individual and developmental differences, and necessary causes. In P.M. Fletcher (Ed.), The handbook of child language (pp. 180-194). Oxford: Blackwell. 